REVISTA

\title{
ODONATOFAUNA EM FLORESTA ESTACIONAL SEMIDECIDUAL MONTANA DO SUL DO ESTADO DE MINAS GERAIS
}

\section{ODONATOFAUNA IN A SEASONAL MONTANE SEMIDECIDUOUS FOREST IN SOUTHERN MINAS GERAIS}

\author{
Giovanna Aparecida Cetra Silva ${ }^{1 *}$ Marcos Magalhães de Souza ${ }^{2}$ \\ ${ }^{1}$ IFSULDEMINAS Campus Inconfidentes, cetragiovanna@gmail.com; \\ ${ }^{2}$ IFSULDEMINAS Campus Inconfidentes, marcos.souza@ifsuldeminas.edu.br \\ *Autor de correspondência
}

Artigo submetido em 04/01/2020, aceito em 14/04/2020 e publicado em 28/08/2020.

Resumo: A ordem Odonata reúne insetos popularmente conhecidos como libélulas, que desempenham importante papel nos ecossistemas dulcícolas. Entretanto, há muitas regiões do Brasil com pouca ou nenhuma informação sobre esses insetos, mesmo em estados considerados bem estudados como Minas Gerais. Nesse aspecto, o presente trabalho objetivou realizar o levantamento da odonatofauna de floresta estacional em municípios da região do Sul do estado de Minas Gerais. O estudo foi realizado no período de dezembro de 2016 a março de 2018, totalizando 40 dias de coleta e 240 horas amostrais. Foram coletados 400 espécimes distribuídos em 71 espécies, 33 gêneros e 10 famílias, sendo as duas mais frequentes a Libellulidae e a Coenagrionidae. A espécie abundante foi Acanthagrion gracile. A área de estudo apresenta elevada riqueza de espécies e é o segundo trabalho a registrar a família Pseudostigmatidae no estado mineiro.

Palavras-chave: Pseudostigmatidae; Acanthagrion; Libellulidae.

\begin{abstract}
The Odonata Order includes insects popularly known as dragonflies, which play an important role in freshwater ecosystems; however there are many regions of Brazil with little or no information about these insects, even in states considered well studied as Minas Gerais. In this aspect, the present work aimed to research the odonatofauna seasonal forest fauna in municipalities of the southern region of the state Minas Gerais. The study was carried out from December 2016 to March 2018, totaling 40 collection days and 240 sample hours. We collected 400 specimens distributed in 71 species, 33 genera and 10 families, Libellulidae and Coenagrionidae being the most frequent families. The most frequent and abundant specie was Acanthagrion gracile. The study area has a high species richness and it is the second work to register the Pseudostigmatidae family in the state of Minas Gerais.
\end{abstract}

Keywords: Pseudostigmatidae; Acanthagrion; Libellulidae. 


\section{INTRODUÇÃO}

A ordem Odonata apresenta cerca de 5.680 espécies, incluindo popularmente os insetos conhecidos no Brasil por donzelinhas ou libélulas. Na fase de ninfa, as libélulas ocupam ecossistemas aquáticos dulcícolas, lênticos ou lóticos, e na adulta alada, os ambientes terrestres associados (KALKMAN et al., 2008).

A ordem é dividida em duas subordens Epiprocta e Zygoptera. (REHN, 2003). No Brasil são conhecidas 860 espécies de odonatos distribuídos em 146 gêneros (PINTO, 2019). Há a presença de 15 famílias, sendo elas Polythoridae, Calopterygidae, Pseudostigmatidae, Dicteriadidae, Lestidae, Coenagrionidae, Perilestidae, Megapodagrionidae, Protoneuridae, Amphipterygidae da subordem zygoptera e Libellulidae, Aeshnidae, Cordullidae e Gomphidae de Epiprocata (SOUZA et al., 2007), segundo Pinto (2019) há ainda a inclusão da família Heteragrionidae dentro da subordem Zygoptera.

Esses insetos possuem quatro asas membranosas com venação densa, abdômen longo e fino, apresentando coloração vibrante. Os adultos apresentam o hábito de voos perto de córregos, rios lagoas e outros corpos d'água doce ou salobra (RAMÍREZ, 2010). Apresentam desenvolvimento hemimetábolos (passando pelos estágios de ovo, ninfa e adultos), depositando seus ovos em substratos submersos, vegetações adjacentes aos corpos de água ou diretamente no ambiente aquático (RAMÍREZ, 2010). A duração do período larval pode variar de dois meses a dois anos, de acordo com cada espécie (SOUZA et al., 2007).

As libélulas são exclusivamente predadoras generalistas, alimentando-se de crustáceos, protozoários, nematóides, diferentes ordens de insetos em fase larval, alevinos, girinos e praticam $\mathrm{o}$ ato de canibalismo. Todavia, servem de alimento para alguns predadores como aves, peixes, anfíbios, répteis (COSTA et al., 2012) e outros insetos como a mosca-assassina da família Asilidae (SOUZA et al., 2017a). As libélulas constituem importantes componentes das cadeias tróficas de diferentes ecossistemas, inclusive sendo benéfico para o homem, uma vez que se alimentam de insetos capazes de transmitir doenças (COSTA et al., 2012).

Algumas espécies de libélulas são consideradas como bioindicadores da qualidade do ambiente em que vivem, uma vez que, em determinadas espécies, suas ninfas têm como requisito algumas condições próprias para que possam permanecer naquele habitat, sendo que alterações bruscas podem levá-las a extinção (COSTA et al., 2012).

O Brasil é o país de maior diversidade de odonata no mundo, entretanto são poucos estudos de inventários, mesmo em estados considerados bem amostrados como Minas Gerais, onde os primeiros estudos de riqueza e distribuição de populações foram realizados por Santos (1970) no município de Itatiaia, região vizinha da sede do atual Parque Nacional de Itatiaia, e Santos (1966), no município de Poços de Caldas ambos no sul do estado.

No período de 2002 a 2019 a literatura registrou 10 estudos, destaque para Bedê et al., (2015), que apresenta a lista de espécies de odonata do Refúgio da Vida Silvestre para Proteção das libélulas. Trata-se da única Unidade de Conservação relacionada à proteção desses insetos no Brasil, localizada no município de Tiradentes, que abriga a maior riqueza de odonata do mundo.

Em alguns trabalhos foram descritos novos gêneros como Franciscobasis e Franciscagrion, além de novas espécies (MACHADO; SOUZA, 2014; MACHADO; BEDÊ 2015; MACHADO, 2015), mas ainda assim há poucas informações e muitos ecossistemas são subamostrados ou não possuem informações (SOUZA et al. 2017b; 
SOUZA et al., 2018).

Dessa forma o presente estudo objetiva realizar o levantamento da odonatofauna em áreas de floresta estacional semidecidual montana associados a ecossistemas dulcícolas lênticos e lóticos em Minas Gerais, e ampliar informações quanto à riqueza e à distribuição das populações de odonata no estado.

\section{MATERIAIS E MÉTODOS}

O estudo foi realizado em fragmentos de florestas estacional semidecidual montana associados a curso d'água nos municípios de Inconfidentes, Ouro Fino e Tocos do Moji, no Sul de Minas Gerais,

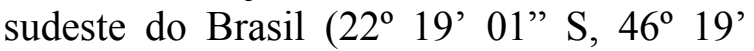
$\left.42^{\prime \prime} \mathrm{W}\right),\left(22^{\circ} 16^{\prime} 59^{\prime \prime} \mathrm{S}, 46^{\circ} 22^{\prime} 08^{\prime \prime} \mathrm{W}\right)$ e

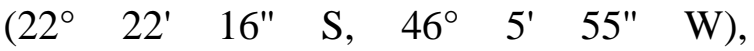
respectivamente.

As coletas foram realizadas em cinco áreas diferentes dos municípios de Inconfidentes, sendo elas denominadas de Fazenda IFSULDEMINAS $\left(22^{\circ} 18^{\prime} 47.41^{\prime \prime S}\right.$, $\left.46^{\circ} 19^{\prime} 57.26^{\prime \prime W}\right)$, Pedreira (22 21'28.38"S, $\left.46^{\circ} 18^{\prime} 59.37^{\prime \prime W}\right)$, Cachoeira dos Magalhães (22 $\left.22^{\prime} 15.73 " \mathrm{~S}, 46^{\circ} 17^{\prime} 59.17^{\prime \prime} \mathrm{W}\right)$,

Monjolinho (22¹9'18.01's, $\left.46^{\circ} 18^{\prime} 23.81^{\prime \prime} \mathrm{W}\right), \quad$ Fragmento florestal 1 $\left(22^{\circ} 20^{\prime} 33.61 " \mathrm{~S}, 46^{\circ} 20^{\prime} 23.88^{\prime \prime W}\right)$, em duas áreas em Ouro Fino, Fragmento florestal 2 $\left(22^{\circ} 21^{\prime} 8.01^{\prime \prime S}, 46^{\circ} 20^{\prime} 21.32^{\prime \prime W}\right)$, Fragmento florestal $3\left(22^{\circ} 22^{\prime} 57.07 " \mathrm{~S}, 46^{\circ} 20^{\prime} 8.86^{\prime \prime W}\right)$ e uma área em Tocos do Moji, hidrelétrica $\left(22^{\circ} 18^{\prime} 37.07^{\prime \prime} \mathrm{S}, 46^{\circ} 12^{\prime} 6.46^{\prime \prime} \mathrm{W}\right)$ (Figura 1). Essas áreas são formadas por fragmentos florestais em diferentes estágios de sucessão ecológica e de conservação, associadas a ambientes lênticos, açudes e lagos, e lóticos como rios e córregos.

As coletas realizadas perfizeram 40 dias, com média de seis horas diárias, totalizando 240 horas de dezembro de 2016 a março de 2018.

Para a captura dos exemplares adultos de odonata utilizou-se o método de busca ativa, com o auxílio de redes entomológicas (puçás), com a metodologia semelhante à de
Bedê e Machado (2002). As coletas ocorreram por meio de caminhadas próximas aos cursos hídricos, tanto lênticos como lóticos, para a captura dos espécimes.

Figura 1: Pontos de coleta da odonatofauna em diferentes áreas nos municípios de Inconfidentes, Ouro Fino e Tocos do Moji, estado de Minas Gerais.

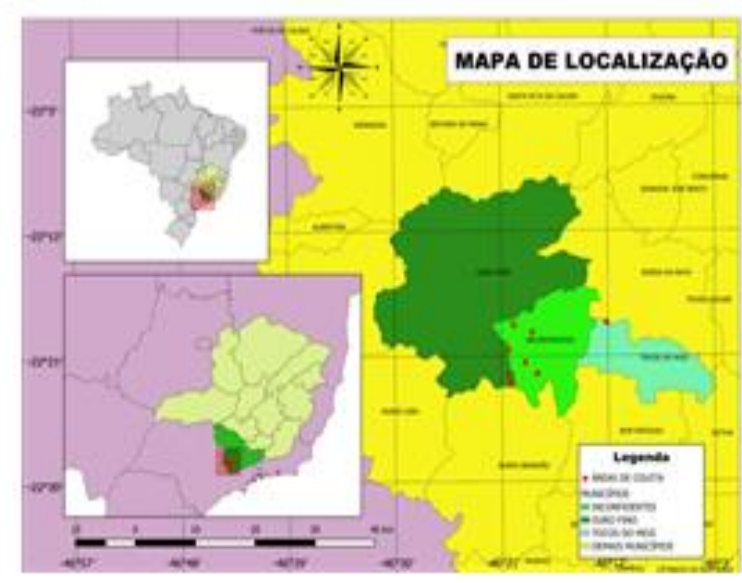

Fonte: Próprio autor (2020).

A busca ativa dos espécimes ocorreu tanto nos períodos matutinos como nos vespertinos, com preferência por dias ensolarados, mas também foram realizadas coletas em dias nublados.

Os espécimes encontrados no campo foram acondicionados em envelopes entomológicos e, após 72 horas, tempo necessário para que houvesse a liberação de excrementos pelos exemplares coletados, foram mergulhados em acetona PA (pura para análise). A acetona dissolve a gordura e auxilia a preservação da coloração (AMORIM et al., 2018). Posteriormente, foram enviados ao professor Dr. Ângelo Parise Pinto, da Universidade Federal do Paraná (UFPR), onde o material foi identificado e armazenado na Coleção daquela universidade.

A riqueza da fauna de Odonata foi comparada pelo método Unweighted PairGruop Method using Arithmetic averages (UPGMA), utilizando coeficiente de similaridade de Jaccard (KREBS, 1999), comparando a odonatofauna dos trabalhos de Souza et al., (2013), Bedê et al., (2015) e Amorim et al., (2018), estudos conduzidos 
em área de mesma fitofisionomia no estado de Minas Gerais.

Para avaliar a eficiência de coleta foi utilizado a média de quatro estimadores de espécies (Jackknife 1), com o uso do programa Software R (R Development Core Team, 2017), com 1000 reamostragens.

\section{RESULTADOS E DISCUSSÃO}

Foram coletados 400 espécimes, distribuídos em 71 espécies, 33 gêneros de 10 famílias (Tabela 01).

As famílias de maior riqueza de espécies foram Libellulidae, com 33 e Coenagrionidae, com 20. Isso era esperado, em função do grande número de espécies descritas dessas famílias no Brasil, 228 e 309, respectivamente (PINTO, 2019). Resultados semelhantes foram encontrados por Santos (1966); Santos (1970); FerreiraPeruquetti \& de Marco Junior (2002); Souza et al. (2013); Bedê et al. (2015); Dos Anjos (2017); Vilela et. al. (2016); Souza et al. (2017b); Amorim et al. (2018); Borges et al. (2019), no estado de Minas Gerais.

O trabalho de Almeida et al., (2013) foi o único que, diferentemente dos demais referentes à diversidades de Minas Gerais, não apresentou Libellulidae e Coenagrionidade como famílias mais abundantes, e sim Libellulidae e Gomphidae. Isso se deve ao fato da utilização de armadilhas luminosas e malaise, uma vez que essa família é difícil de ser capturada pelo método tradicional de busca ativa por rede entomológica (ALMEIDA et al., 2013).
Apesar de o local de estudo localizar-se a cerca de $25 \mathrm{~km}$ da área estudada por Amorim et al., (2018), no presente trabalho, 24 espécies e uma família não foram registradas (Tabela 1).

Isso, em parte, é reflexo do trabalho de Amorim et al. (2018) ter se concentrado em ambientes lóticos, sobretudo em cachoeiras, enquanto este realizou maior amostragem em ambientes lênticos, como açudes, que segundo Hanauer et al. (2014), caracterizam-se por serem ecossistemas antropizados, e que comportam espécies com ampla valência ecológica (JUEN et al., 2014), o que explica a ocorrência de espécies distintas no presente trabalho, quando comparado ao de Amorim et al., 2018.

Outro fator que poderia explicar a diferença da odonatofauna entre os dois estudos seria a altitude, que difere cerca de 300 metros entre as duas áreas.

Sabe-se que essa variável ambiental afeta a composição das comunidades de invertebrados aquáticos (TOMANOVA et al., 2007). Entretanto, segundo Corbet (2004), esse fator não afeta algumas espécies, principalmente aquelas de grande capacidade voo.

De acordo com Oliveira-Junior et al., (2015), o fator altitude isoladamente não afeta odonata, mas se associado a outras variáveis ambientais, como grau de conservação da área e características físicoquímica da água, afetaria a composição da fauna. Contudo, o estudo de Oliveira-junior et al., (2015) se ateve a igarapés na 
Tabela 01: Famílias, espécies e número de indivíduos de odonata coletadas em floresta estacional semidecidual montana nos municípios de Inconfidentes, Ouro Fino e Tocos do Moji, de dezembro de 2016 a março de 2018

\begin{tabular}{|c|c|}
\hline Família/Espécie & $\begin{array}{c}\text { No. de } \\
\text { indivíduos }\end{array}$ \\
\hline Aeshinidae & \\
\hline Anax concolor Brauer, 1865 & 02 \\
\hline Gomphidae & \\
\hline Aphila teodorina (Navás, 1933) & 01 \\
\hline Progomphus complicatus Selys, 1854 & 01 \\
\hline Libellulidae & \\
\hline Brachymesia furcata (Hagen, 1861) & 03 \\
\hline Brechmorhoga nubecula Rambur, 1842 & 04 \\
\hline Dasythemis mincki mincki (Karsch, 1889) & 11 \\
\hline Dythemis nigra Martin, 1897 & 05 \\
\hline Elasmothemis constricta (Calvert, 1898) & 01 \\
\hline Erythemis attala (Selys in Sagra, 1857) & 01 \\
\hline Erythrodiplax fusca (Rambur, 1842) & 22 \\
\hline Erythrodiplax juliana Ris, 1911 & 11 \\
\hline Erythrodiplax latimaculata Ris, 1911 & 02 \\
\hline Erythrodiplax media Borror, 1942 & 17 \\
\hline Erythrodiplax melanorubra Borror, 1942 & 01 \\
\hline Erythrodiplax paraguayensis (Forster, 1904) & 02 \\
\hline Erythrodiplax sp. & 06 \\
\hline Macrothemis heteronycha (Calvert, 1909) & 01 \\
\hline Macrothemis imitans imitans Karsch, 1890 & 08 \\
\hline Macrothemis musiva Calvert, 1898 & 01 \\
\hline Macrothemis tenuis Hagen, 1868 & 04 \\
\hline Miathyria marcella (Selys in Sagra 1857) & 02 \\
\hline Micrathyria almeidai Santos, 1945 & 01 \\
\hline Micrathyria didyma (Selys in Sagra, 1857) & 01 \\
\hline Micrathyria hesperis Ris, 1911 & 10 \\
\hline Micrathyria hipodidyma Calvert, 1906 & 01 \\
\hline Micrathyria laevigata Calvert, 1909 & 01 \\
\hline Micrathyria ocellata dentiens Calvert, 1909 & 02 \\
\hline Nephepeltia berlai Santos, 1950 & 03 \\
\hline Oligoclada laetitia Ris, 1911 & 02 \\
\hline Orthemis cultriformis Calvert, 1909 & 01 \\
\hline Orthemis discolor Burmeister, 1839 & 07 \\
\hline Pantala flavescens (Fabricius, 1798) & 01 \\
\hline Perithemis icteroptera (Selys, 1857) & 03 \\
\hline Perithemis lais (Perty, 1834) & 01 \\
\hline Perithemis mooma Kirby, 1889 & 03 \\
\hline Tramea binotata (Rambur, 1842) & 01 \\
\hline $\begin{array}{l}\text { Calopterygidae } \\
\text { Hetaerina longipes Hagen, } 1853\end{array}$ & 08 \\
\hline
\end{tabular}


\begin{tabular}{l|l}
\hline Hetaerina proxima Selys, 1853 & 10
\end{tabular}

Hetaerina rosea Selys, $1853 \quad 05$

Hetaerina simplex Selys, $1853 \quad 01$

Hetaerina sp. 1

\begin{tabular}{l|l} 
Mnesarete guttifera (Selys, 1873) & 05
\end{tabular}

Coenagrionidae

Acanthagrion aeopilum Tennessen, 2004

Acanthagrion gracile (Rambur, 1842)

Acanthagrion lancea Selys, 1876

Argia lilacina Selys, 1865

Argia modesta Selys, $1865 \quad 06$

Argia mollis Hagen, 1865

Argia sordida Hagen, $1865 \quad 06$

Argia sp. 1

Argia sp. 2

Homeoura chelifera (Selys, 1876) 12

Ischnura capreolus (Hagen, 1861)

Ischnura fluviatilis Selys, $1876 \quad 01$

Oxyagrion basale Selys, $1876 \quad 09$

Oxyagrion chapadense Costa, 1978

$\begin{array}{ll}\text { Oxyagrion } \mathrm{sp} .1 & 07\end{array}$

Oxyagrion sp.2 02

Oxyagrion terminale Selys, $1876 \quad 23$

Telebasis carmesina Calvert, $1909 \quad 01$

\begin{tabular}{l|l} 
Telebasis griffinii (Martin, 1896) & 04
\end{tabular}

\begin{tabular}{l|l} 
Telebasis willinki Fraser, 1948 & 14
\end{tabular}

\begin{tabular}{l|l} 
Heteragrionidae & 14 \\
\hline
\end{tabular}

Heteragrion aurantiacum Selys, $1862 \quad 01$

Lestidae

\begin{tabular}{l|l} 
Archilestes exoletus (Hagen, 1862) & 02
\end{tabular}

Lestes dichrostigma Calvert, $1909 \quad 01$

Lestes forficula Rambur, $1842 \quad 03$

Lestes paulistus Calvert, $1909 \quad 02$

\begin{tabular}{l|l} 
Lestes $\mathrm{sp}$. & 01
\end{tabular}

\begin{tabular}{l|c}
\hline $\begin{array}{l}\text { Megapodagrionidae } \\
\text { Allopodagrion contortum (Hagen in Selys, 1862) }\end{array}$ & 03 \\
\hline $\begin{array}{l}\text { Protoneuridae } \\
\text { Neoneura sylvatica Hagen in Selys, 1886 }\end{array}$ & 01 \\
\hline Pseudostigmatidae & 04 \\
Mecistogaster mielkei Machado e Lacerda, 2017
\end{tabular}

Fonte: Próprio autor (2020). 
Tabela 2: Relação dos trabalhos de inventários de Odonata em Minas Gerais.

\begin{tabular}{cc}
\hline Autores e data do estudo & Espécies e famílias \\
\hline Bedê et al., 2015 & 128 espécies e 10 famílias \\
Presente estudo & 071 espécies e 10 famílias \\
Amorim et al., 2018 & 071 espécies e 08 famílias \\
Dos Anjos, 2016 & 068 espécies e 11 famílias \\
Santos, 1970 & 060 espécies e 10 famílias \\
Santos, 1966 & 059 espécies e 09 famílias \\
Souza et al., 2013 & 057 espécies e 09 famílias \\
Borges et al., 2019 & 036 espécies e 06 famílias \\
Vilela et al., 2016 & 031 espécies e 05 famílias \\
Souza et al., 2017b & 048 espécies e 07 famílias \\
Ferreira-Peruquetti \& de Marco Júnior, 2002 & 028 espécies e 07 famílias \\
Almeida et al., 2013 & 026 espécies e 06 famílias \\
\hline
\end{tabular}

Fonte: Próprio autor (2020).

Amazônia com variações altimétricas de 120 metros, o que, portanto, não descarta a diferença de altitude ter afetado a composição da odonatofauna discutida no presente trabalho.

Considerando o exposto, áreas próximas geograficamente podem abrigar comunidades distintas de libélulas, e justificam estudos de biodiversidade nessas condições.

Este estudo apresenta-se como o segundo mais rico em odonatofauna no estado de Minas Gerais (Tabela 2), devido ao trabalho amostral realizado em horas e dias para a captura das espécies, quando comparado a outros estudos. Entretanto, o número pode ser ainda maior, como mostra o estimador de riqueza de espécies (80 spp.) pelo teste jackknife (Figura 2), indicando $89 \%$ de eficiência amostral.

Merece destaque o registro da família Pseudostigmatidae, que ocorreu em estudos de diversidade somente no trabalho de Santos (1970). As espécies registradas por Santos foram Mecistogaster amalia (Burneister, 1839) e Mecistogaster austictus, Selys, 1860. No presente trabalho, registrou-se a ocorrência de Mecistogaster mielkei, recentemente descrita por Lacerda (2017), (Figura 3). A família Pseudostigmatidae é pouco encontrada, devido ao hábito fitotelmo, que consiste em realizar a postura de ovos em cavidades de árvores e em plantas como as bromélias ou ocos de bambu, locais onde armazenam-se água da chuva (FINCKE, 2005).

A análise de similaridade de fauna (Figura 5) revelou maior proximidade com o trabalho de Amorim et al., (2018), que foi realizado no município de Bueno Brandão. Isso se deve ao fato das duas localidades encontrarem-se próximas geograficamente, uma vez que todos os trabalhos submetidos a esse teste apresentam a mesma fitofisionomia. A exceção é o trabalho de Bedê et al. (2015), que apresenta diferentes ecossistemas associados à floresta semidecidual, como campo rupestre e enclaves de Cerrado. Tal fato ajudaria a entender a baixa similaridade (Figura 05). 
Gráfico 1: Curva de acúmulo de espécies pelo estimador de riqueza Jackknife, indicando um número em torno de 80 espécies de odonata para região do estudo, comparado com as 71 coletadas.

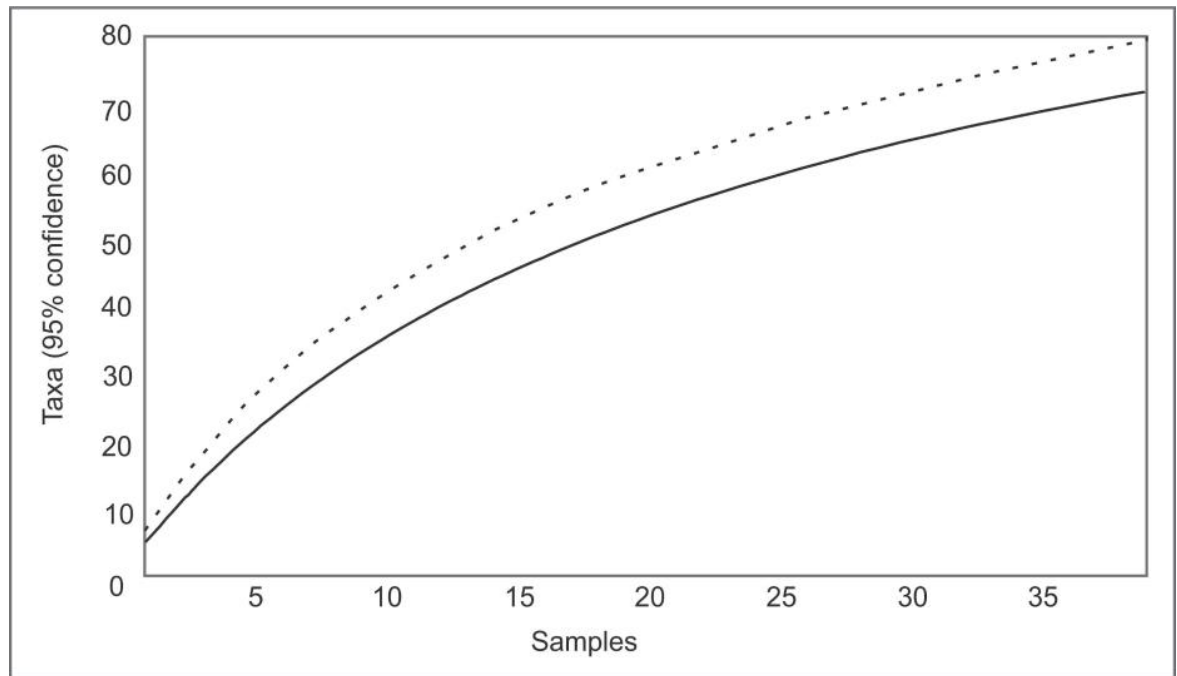

Fonte: Próprio autor (2020).

Figura 3: Espécie Acanthagrion gracile, na área de estudo.

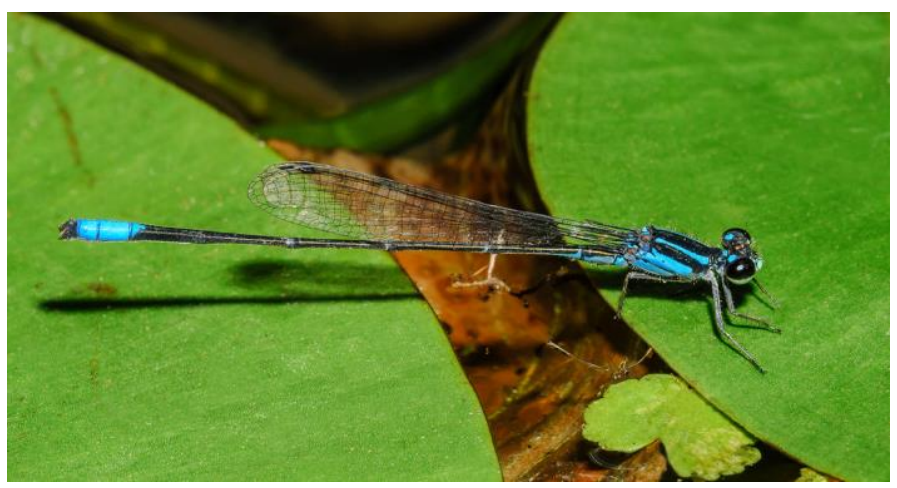

Fonte:https://hiveminer.com/Tags/neotropical\%2Czygoptera.

Figura 4: Espécie de Mecistogaster mielkei encontrada no município de Inconfidentes, sul do estado de Minas Gerais.

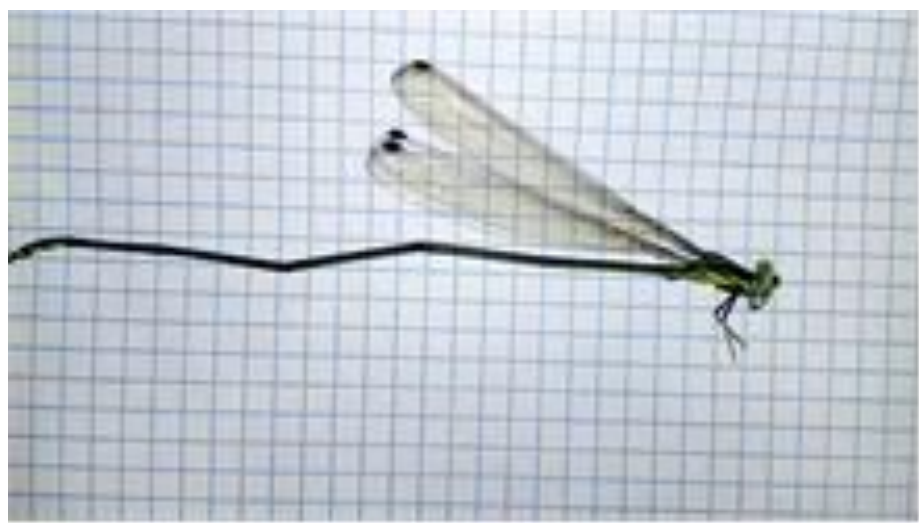

Fonte: Próprio autor (2020). 
Figura 5: Similaridade da fauna de odonata do presente estudo com os trabalhos de Souza et al., 2013; Bedê et al., 2015 e Amorim et al., 2018.

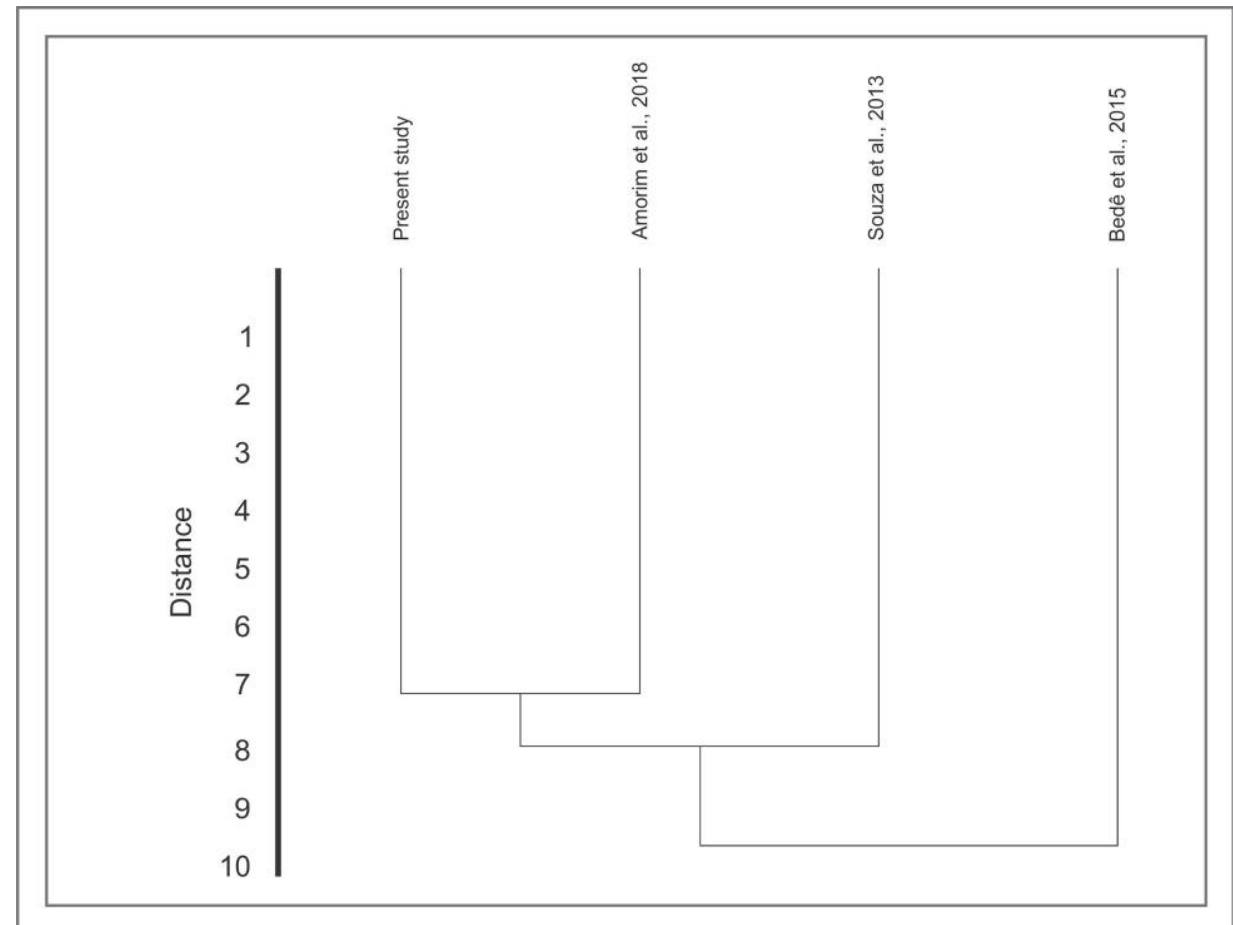

Fonte: Próprio autor (2020).

\section{CONCLUSÕES}

O trabalho mostrou ser uma importante ferramenta para mensurar a riqueza de odonata no Sul do estado de Minas Gerais e elucidou o potencial biológico encontrado nos municípios amostrados. A presença da espécie Mecistogaster mielkei, família Pseudostigmatidae, evidencia a importância da conservação dessas áreas para a proteção da odonatofauna no referido estado. Por fim, revelou que estudos em áreas geográficas próximas, mas que apresentam variáveis ambientais distintas, possuem comunidades diferentes de odonata, o que reforça que pequenas alterações no ambiente podem influenciar diretamente na riqueza de espécies.

\section{AGRADECIMENTOS}

Ao Dr. Ângelo Parise Pinto (UFPR) pelas identificações. Ao Instituto Federal de Educação, Ciência e Tecnologia do Sul de
Minas, Campus Inconfidentes pela logística; ao Dr. Mateus Aparecido Clemente pelas análises; e aos alunos do laboratório de zoologia pelo auxílio nas coletas.

\section{REFERÊNCIAS}

ALMEIDA, M. V. O.; PINTO, A. P.;
CARVALHO, A. L.; TAKIYA, D. M. When rare is just a matter of sampling: Unexpected dominance of clubtail dragonflies (Odonata, Gomphidae) through different collecting methods at Parque Nacional da Serra do Cipó, Minas Gerais State, Brazil. Revista Brasileira de Entomologia, v. 57, n. 4, p. 417-423, 2013. AMORIM, M. S.; SOUZA, M. M.; DOS ANJOS, C.S. Riquezas de libélulas (Insecta: Odonata) no município de Bueno Brandão, sul de Minas Gerais. Revista MG Biota, Belo Horizonte, v.11, n.1, p.16-32, 2018. BORGES, L. R.; BARBOSA, M.S.; CARNEIRO, M. A.A.; VILELA, D.S.; SANTOS, J. C. Dragonflies and damselflies 
(Insecta: Odonata) from a Cerrado area at Triângulo Mineiro, Minas Gerais, Brazil.

Revista Biotaneotropica, v.19, n.1, p. e20180609, 2019.

BEDÊ, L. C.; MACHADO, A. B. M.

Diagnóstico da condição ambiental em ambientes úmidos na região do Parque Nacional da Serra da Canastra-MG utilizando libélulas como indicadores ecológicos. Relatório final - Consórcio da Usina Hidrelétrica de Igarapava, 36 p., 2002.

BEDÊ, L. C.; MACHADO, A. B. M.; PIPER, W.; SOUZA, M. M. Odonata of the Serra de São José - Brazil's first Wildlife Reserve aimed at the conservation of dragonflies. Notulae odonatologicae, v. 8 , n. 5, p. 117-128, 2015.

CORBET, P. S. Dragonflies: behaviour and ecology of Odonata. Colchester, Essex, England: Harley Books, c.1999. 829p, 2004. COSTA, J. M., SANTOS, T. C., OLDRINI, B. B., Insetos do Brasil: Diversidade e Taxonomia; In: RAFAEL, J. A., MELO, G. A. R., de CARVALHO, C. J. B., CASARI, S.A., CONSTANTINO. R; Odonatas; Ribeirão Preto: Holos Editora; Cap. 18; p. 245-256, 2012.

DOS ANJOS, C. S. Riqueza de odonatofauna (insecta) em floresta mista no estado de Minas Gerais, Brasil. 2017. 26p. Trabalho de Conclusão de Curso (Licenciatura em Ciências Biológicas) Instituto Federal de Educação, Ciência e Tecnologia do Sul de Minas Gerais. FERREIRA-PERUQUETTI, P. S.; DE MARCO JR, P. Efeito da alteração ambiental sobre comunidades de Odonata em riachos de Mata Atlântica de Minas Gerais, Brasil. Revista brasileira de Zoologia, v. 19, n. 2, p. 317-327, 2002. FINCKE, O. M. Use of Forest and Tree Species, and Dispersal by Giant Damselflies (Pseudostigmatidae): Their Prospects in Fragmented Forests. In: RIVERA, A. C. Fourth WDA International Symposium of Odonatology. Pontevedra (Spain). Sofia Moscow: Pensoft Publishers, pp. 103-125, 2005.
HANAUER, G.; RENNER, S.; PERICO, E. Inventariamento Preliminar da Fauna de Libélulas (Odonata) em quatro municípios do Vale Taquari/RS. Revista Destaques Acadêmicos, v. 6, n. 3, 2014. JUEN, L.; OLIVEIRA JUNIOR, J. M. B.; SHIMANO, Y.; CABETTE, H. S. R. Composição e riqueza de Odonata (Insecta) em riachos com diferentes níveis de conservação em um ecótone CerradoFloresta Amazônica. Acta Amazônica, v. 44, n. 2, p. 175-184, 2014.

KALKMAN, V. J.; CLAUSNITZER, V.; DIJKSTRA, K. D. B.; ORR, A. G.; PAULSON, D. R \& VAN TOL, J. Global diversity of dragonflies (Odonata) in freshwater. Hydrobiologia, v. 198, p.351363, 2008. DOI:

https://doi.org/10.1007/978-1-4020-8259738

KREBS, C. J. Ecological Methodology. 2 ed. New York: Benjamin/ Cummings, 620p, 1999.

LACERDA, D. S. S. Estudo taxonômico de Mecistogaster Rambur,1842 e Platystigma Kennedy, 1920 da Mata Atlântica (Odonata: Pseudostigmatidae). 2017. P. 103. Tese (Doutorado em Zoologia) - Universidade Federal de Minas Gerais

MACHADO, A. B. M.; SOUZA, M. M. A remarkable new species of Heteragrion from Brazil (Odonata: Megapodagrionidae). International Journal Of Odonatology, v.17, n. 2-3, p.95-99, 2014. DOI: https://doi.org/10.1080/13887890.2014.925 $\underline{514}$

MACHADO, A. B. M. Heteragrion thais sp. nov. from the Atlantic Forest of Brazil (Odonata: Heteragrionidae).

Odonatologica, v. 44, n. 3. p. 391-396, 2015.

MACHADO, A. B. M.; BEDÊ, L. C. Two new genera and nine new species of damselflies from a localized area in Minas Gerais, Brazil (Odonata: Zygoptera). International Journal Of Odonatology, v. 18, n. 4, p.269-296, 2015.

OLIVEIRA-JUNIOR, J. M. B.; SHIMANO, Y.; GARDNER, T.A.; 
HUGHES, R. M.; DE MARCO JÚNIOR, P.; JUEN, L.; Neotropical dragonflies (Insecta: Odonata) as indicators of ecological condition of small streams in the eastern Amazon. Australegology, v.40, p. 733-744, 2015

PINTO, A. P. Odonata in Catálogo

Taxonômico da Fauna do Brasil. 2019.

PNUD. Disponível em:

$<$ http://fauna.jbrj.gov.br/fauna/faunadobrasi

1/171>. Acesso em: 20 fev. 2019.

RAMÍREZ, A. Odonata. Capítulo 5.

Instituto para Estudios de Ecosistemas

Tropicales, Universidad de Puerto Rico,

Revista Biol. Tropical. v. 58, n.4 p. 97 -

136. 2010. Disponível em:

$<$ https://revistas.ucr.ac.cr/index.php/rbt/artic le/view/20084/20286>. Acesso em: 15 nov. 2018.

REHN, A. C. Phylogenetic analysis of higher-level relationships of Odonata.

Systematic Entomology, v.28, n.2, p.181240, 2003. DOI:

https://doi.org/10.1046/j.1365-

3113.2003.00210.x

RENNER, S. Composição de libélulas

(Odonata) em diferentes ambientes da

floresta Nacional de São Francisco de

Paula, Rio Grande do Sul, Brasil. 2014.

58p. Dissertação (Mestrado em Ambiente e

Desenvolvimento) - Centro Universitário

Univates.

SANTOS, N. D. Odonatos da região de Poços de Caldas, Minas Gerais. Atlas da

Sociedade de Biologia do Rio de Janeiro, do Rio de Janeiro, n. 10, p. 65-68, 1966.

SANTOS, N. D. Odonatos de Itatiaia

(Estado do Rio de Janeiro) da Coleção

Zikan, do Instituto Oswaldo Cruz. Atlas da

Sociedade de Biologia do Rio de Janeiro,

Rio de Janeiro, n. 13, p. 203-205, 1970.

SOUZA, L. O. I, COSTA, J. M \&

OLDRINI, B. B. 2007. Odonata. In: Guia

on-line: Identificação de larvas de Insetos

Aquáticos do Estado de São Paulo.2007

Froehlich, C.G. (org). Disponível em:

$<$ http://sites.ffclrp.usp.br/aguadoce/Guia_on

line/Guia_online_Odonata_Vers\%C3\%A3o

1\%C3\%9F2.0.pdf> Acesso em: 17 nov.

2018.
SOUZA, M. M.; SOUZA, B.; PEREIRA, M. C. S. A.; MACHADO, A. B. M. List of Odonata from Mata do Baú, Barroso, Minas Gerais, Brazil. Journal of species lists and distribution Check List, v. 9, n. 6, p. 1367-1370, 2013. DOI: http://dx.doi.org/10.15560/9.6.1367 SOUZA, M. M.; DOS ANJOS, C. S.; MILANI, L. R.; BRUNISMANN, A. G. Libélulas (Odonata) predadas por moscasassassinas (Diptera: Asilidae) no estado de Minas Gerais, sudeste do Brasil. Revista Brasileira de Zoociências, Juiz de Fora, v.19, n.1, p. e-2596-3325. 2017a DOI: https://doi.org/10.34019/2596-

3325.2018.v19.24709

SOUZA, M. M.; PIRES, E. P.; BRUNISMANN, A. G.; MILANI, L, R.; PINTO, A. P. Dragon ies and damsel ies (Odonata) from the wetland of the Rio Pandeiros, northern region of Minas Gerais State, Brazil, with a description of the male of Archaeogomphus vanbrinki Machado (Anisoptera: Gomphidae). International Journal of Odonatology, v. 20, n. 1, p.1326, 2017b.

SOUZA, M. M.; DOS ANJOS, C. S.; BRUNISMANN, A. G.; MILANI, L. R. Distribuição e riqueza de Heteragrionidae (Odonata) por ecossistema no estado de Minas Gerais. Revista MG Biota, Belo Horizonte, v. 11, p.22-31, 2018.

TOMANOVA, S.; TEDESCO, P. A.; CAMPERO, M.; VAN DAMME, P. A.; MOYA, N.; OBERDORFF, T. Longitudinal and altitudinal changes of macroinvertebrate functional feeding groups in neotropical streams: a test of the River Continuum Concept. Fundamental and Applied Limnology/Archiv für Hydrobiologie, v. 170, n. 3, p. 233-241, 2007.

VILELA, D. S.; FERREIRA, R. G.; DELCLARO, K. The Odonata community of a brazilian vereda: seasonal patterns, species diversity and rarity in a palm swamp environment. Bioscience Journal, Uberlândia, v. 32, n. 2, p. 486-495, 2016. DOI: https://doi.org/10.14393/BJv32n2a2016-30491. 